\title{
Extending the role of glossaries in a virtual learning environment
}

\author{
Katherine Sinitsa and Alla Manako \\ International Research and Training Center of Information Technologies and Systems \\ UNESCO/IIP, 40, prosp. Glushkova, 252650,Kiev, Ukraine.kath@umod.kiev.ua
}

Keywords: learning environment, cognitive tools, hyper-glossary, autonomous learner

\begin{abstract}
A virtual learning environment comprises of a combination of tools that facilitate learning experience. For an autonomous learner, it is crucial that those tools provide cognitive support necessary to arrange, plan, monitor and assess learning on one's own. This paper offers some ways to reconstruct a glossary into a cognitive tool and describes its application in autonomous learning.
\end{abstract}

\section{INTRODUCTION}

The technological revolution in education is accompanied by the changes of the research focus from instructional strategies to learning support tools, from a teacher-centred to a learner-centred paradigm and further to a learning-centred one (Boy 1996). The most recent approaches to learning with an emphasis on cognitive function, provide a common platform for the educational technologies by addressing both children and adults as lifelong learners.

Within this framework, the focus of educational software design shifts from courseware to learning environments, which are aimed at the facilitation of knowledge and skills acquisition, exploration of information resources represented by the different media, support of hands-on experiences (learning by doing), and other learning activities. A combination of tools available to a learner via telecommunication facilities forms a virtual learning environment (VLE), which 'enable the full 
information, exploration, communication and feedback, as if you are in a well-organised classroom.' (Kommers and Zhiming 1998).

VLE opens new horizons in learning and training, and a variety of approaches and implementations are still waiting for systematisation and classification. We will focus further in this submission on learning environments for an autonomous learner, that is, we will not consider collaborative learning, or instructor's guidance and support. In contrast to the instructional design, which arranges both content and control of learning, an environment for an autonomous learner should support a learning cycle defined and managed by a learner themself.

We consider an 'autonomous learner' as a person seeking additional information, trying to enlarge their knowledge, skills or capabilities for successful professional activity. Thus, learning needs and requirements are defined by learners themselves, who must also:

- determine the current status of their knowledge in relation to a task;

- set some learning objectives, describing the amount of the content that should be learned, procedures that should be mastered;

- find necessary information sources, tools, services;

- select appropriate units for learning, taking into account both external (technical, economic) and internal (learning style) preferences and restrictions;

- arrange activities to practice in application of the newly acquired knowledge;

- monitor, control and sequence learning;

- master new knowledge and incorporate it into mental model; assess their own understanding.

VLE, organised as a set of inter-operable and communicating systems, may add value to each of its components by the integration and extension of their roles in a learning process and the introduction of cognitive support for a learner. This issue is further explored in more detail with the production of a glossary, which historically is considered in education as a complementary tool for storage and retrieval of short factual information.

The introduction of the hyper-text technology for navigation along the cross-references in a glossary does not change the overall attitude. For the domains with an extensive terminological base, a glossary naturally plays a more important role and can be turned into a support tool for self-learning and self-assessment.

In the following sections we will consider some ways to turn a hyperglossary into an intelligent tool and propose some narratives of the new roles of a hyper-glossary in a learning environment for an autonomous learner. 


\section{COGNITIVE SUPPORT FOR THE AUTONOMOUS LEARNING}

In a classroom learning environment, a tutor keeps the balance between new and known components, defines granularity of learning units, plans, schedules, and monitors a learner's activity, thus allowing a learner to focus on the subject matter. An autonomous learner has to perform both roles simultaneously, which requires reasoning at both subject level and metalevel (control and planning). Their main activities include:

- setting learning goals;

- navigation in the information space and search for relevant information;

- selection and sequencing of the information units;

- mastering separate units;

- assimilation of newly acquired knowledge, its integration into the existed mental structure, update and modification of the structure;

- monitoring their own progress and self-assessment.

To facilitate effective learning, a VLE should provide cognitive support for all of the mentioned activities. Cognitive tools empower learner's mental abilities, engage them in an active creation of the new knowledge structures, and facilitate reflection and focusing (Kommers, Jonassen, and Mayes 1991). In domains containing extensive factual information, such as natural sciences, medicine and some engineering disciplines (such as telecommunications, Internet), the main cognitive load is put on search and combination of the information pieces.

The role of a glossary can be naturally extended from an auxiliary information storage to an intelligent assistant, which keeps the knowledge of the particular details and is able to share it with the user on an indirect request, to communicate or demonstrate the structure of its knowledge, and to assist in search of a specific portion of data. By the indirect request we mean the absence of the unique indication of the information portion that may be relevant. A hyper-glossary empowers the user's ability to grasp a new concept by the well-structured description, convenient interface and comprehensive framework for presentation of the conceptual units in the relation to each other.

A concept-based hyper-glossary with extended visualisation can be considered as a reflection of the conceptual structure of the corresponding domain. It can serve as a backbone for the autonomous learning by support of the following activities of the learner:

- search of information (by providing explanation of selected term, assistance in search of an adequate name for a concept);

- local learning (by the presentation of the structured information about a concept, the features of the correspondent entities, its use.); 
- integration of knowledge (by the opportunity to compare the concepts within a class, to trace the relations between the concepts, to explore the related concepts);

- self-assessment in terminology and structural knowledge of the domain (by the construction of generic tasks over structured glossary entries);

- navigation and planning of learning (by the elaborated visualisation).

In the above description, an analogy can be traced with the concept maps (Novak 1990) that were suggested as support tools for knowledge synthesis and navigation, facilitators and mediators of knowledge exchange, and a hypermedia authoring framework. In contrast to a concept map, which contains units of any size linked to each other by arbitrary labelled relations, a hyper-glossary contains units corresponding to the terms that are linked by the pre-defined relations. These restrictions and its specific structure extend potential applications of a hyper-glossary by adding new functions - the search of a concept name and self-assessment support.

\section{A HYPER-GLOSSARY IN LEARNING}

A hyper-glossary content is represented as a network of information nodes connected by the links of various types. Some of these links are directed, others are not. A node corresponds to a traditional glossary entry, though its content is structured according to more fields, for instance, 'used', 'structure', 'more specific' are added (Sinitsa and Mizoguchi 1998). Therefore, a hyper-glossary contains two types of information: an explanation of each term, and a description of an overall conceptual structure, which can be retrieved either as a text or a graph. In the text mode, a user can access data about the selected concept only, References to the related concepts are highlighted as hyper-links, and a type of relation between the current concept and a concept related to it is specified by a name of the corresponding description field. This is a basic mode, which provides a traditional view of the glossary content. In a neighbourhood mode one can see concepts related to a chosen one on a kind of a map. A user can browse the conceptual vicinity of a term along the links, getting more detailed information in the supplementary text-mode windows.

\subsection{Local learning}

Local learning is ensured by the hyper-glossary representation as a collection of entries with layered structured information. Layers correspond to the definition, contextual description (related concepts), explanations in various levels of details, references to other electronic sources. An elaborated structure of the hyper-glossary entry complemented by a layered 
content enables a user to extract appropriate information according to their preferences, qualification and a purpose of information retrieval. It also allows them to choose an appropriate level of detail, definition style, presentation mode, visualise conceptual network corresponding to the glossary terms, and to obtain references to further instructional sources.

\subsection{Integrating knowledge}

A hyper-glossary presents not only isolated concepts, but also their links and combinations, providing a 'stained glass view' to the domain. Each unit of information has its own value and at the same time contributes to the reflection of the whole picture. Therefore, both glossary content and structure affects its application as a knowledge acquisition support tool. The representation of a hyper-glossary as a networked structure, connected by the links of several types, facilitates the integration of learning content, allows them to place acquired pieces into the structure, to connect them to already known concepts, trace relations between concepts, compare them, reveal common features, and study their use in particular context. Conceptual structure of the domain reflected in the hyper-glossary forms a skeleton for combination of the extended multimedia learning content around the basic concepts (Elliott, Jones, and Barker 1996).

\subsection{Search of information}

Traditional syntactic search (manual search in a glossary index and automatic search of an exact term) is enriched in the hyper-glossary by approximate syntactic search. Approximate search means an extraction of syntactically related terms and is useful both in case of successful exact search and in a situation when the requested term is not found. Syntactically-related terms in a glossary often belong to some concept hierarchy, enabling a user to trace more general, more specific or 'sister' concepts for the chosen one. Failure of the exact search can be caused by a misprint, a spelling error or an incorrect extraction of the term. Approximate search then offers a user a list of similar terms obtained by partial matching with the entries in a glossary index. They may be, for instance, some terms with a common root: test, testing. This function for separate words is incorporated in the spell-checkers, it can be further elaborated to treat collocations. Its value for the glossary depends on the structure of domain terminology.

More elaborated semantic search helps a user to find a term (a concept name) by its tentative description. Unlike the approximate search, which can be implemented in hyper-glossary with isolated entries, semantic search algorithms require special representation of the hyper-glossary content as a 
network structure with the multi-sorted links between nodes. For semantic search, a notion of a 'neighbourhood' of a concept is introduced. The neighbourhood comprises of concepts that are most tightly related to the chosen one. These concepts are distributed along some 'dimensions', or belong to categories describing the common context, for instance, 'communication protocols' or 'web page design'. The most specific or concrete concepts have neighbourhoods that are rather narrow (consist of a few concepts) and 'plain' (have few dimensions), which is a reflection of the restricted number of contexts in which the term can be used. More general (and more vague) concepts have many more facets and sometimes require terms to be ordered within a certain context to show conceptual distance.

A search of an unknown concept from its description starts with the definition of the terms belonging to its neighbourhood and contexts in which it can be used. In the simplest case, tracing is executed among descendants of the known generic concept(s) of the target concept. Semantic search for the inexperienced glossary users can be guided by a system, for instance, by the introduction of the main categories represented in a hyper-glossary and prompting a user to select appropriate one(s).

Suppose a user is looking for the name of the communication protocol used for a certain type of information exchange. In this case, a generic concept is a 'protocol'. A description of the generic concept defines an 'upper bound' for search of the required concept, i.e., it may be found among the descendants of this concept. For 'protocol', the number of descendants may still be too large for manual search, so additional information about the conceptual neighbourhood may be helpful to cut search space until the number of candidates becomes reasonable for browsing.

Another option to facilitate concept searching is to ask a user for a couple of Keywords, which are related to the target term or describe it. These concepts can be considered as a user's description of the concept neighbourhood that defines some 'boarders' for browsing. If a user is unable to name the appropriate concepts, a service engine may offer some candidates for them to mark as 'relevant' or 'irrelevant'. This keywordbased search is similar to the syntactic keyword matching. The difference is in a way of treating the Keywords, which are considered as the semantically connected concepts rather than syntactic units.

\subsection{Self-assessment}

Approximate syntactic search may not only prevent possible lost of motivation caused by the search failures but also facilitate an active 
knowledge construction, as well as may be used in a self-assessment. To check their understanding, a learner can compose their own definition of some glossary term using examples of the corresponding concept, a description of features of the related (in particular, generic) concepts, and other partial information. The user-constructed definition can be then compared with the actual entry, revealing discrepancies important for planning of further learning.

Besides the above, a number of other 'hyper-glossary reconstruction' tasks can be performed: to draw a conceptual structure (network) for selected glossary entries; to name a concept by its definition; and to group related concepts or to list concepts from the vicinity of a given one. They can be combined with typical exercises to reinforce conceptual knowledge, to recognise examples of the concepts, to distinguish concepts, and to classify objects according to given features. All these activities are based on the specific internal structure of a hyper-glossary and elaborated functions intended to support learning. Assessment and evaluation is done by a learner and is aimed at providing a feedback on learning results.

\subsection{Navigation}

The network representation facilitates natural navigation along the links of the chosen type, and directed search via hierarchical layering of concepts. Semantic links between the glossary terms support both user-guided and system-guided browsing. One can distinguish two types of browsing: a vertical one (along a hierarchy of concepts) and a context one (within a neighbourhood). Exploring hierarchical relations, a user is travelling along 'a kind of' or 'part-whole' links, exploring more general or more specific concepts. At any moment a user's view is focused on some current concept. In the vertical browsing, all concepts situated one level above and one level below along the chosen link are displayed. Selecting one of them, a user changes their focus of attention and sees a picture from a new viewpoint.

In browsing a neighbourhood, a user explores a group of the related concepts by choosing a specific context. New facets of the current concept are revealed by moving from one context to another. A user can further explore any of the related concepts by selecting it as the current one. Visualisation of the information structure and opportunity to follow links of a certain type enables better orientation of a learner in information space and facilitates individual planning of learning, allows them to master content from the most specific concepts to the general ones or backward, to browse a contextual vicinity of the selected concept, and when necessary to study examples. 


\section{BASIC INTERNET GLOSSARY AS A BACKBONE FOR RESOURCES COLLECTION}

As a part of the first distance course on Basics of the Internet run in 1997, a questionnaire was offered to the participants, which contained a self-evaluation and reflection part. The members of the course were diverse in age, educational background, current professional activity, acquaintance with computers and learning goals. Information extracted from 342 questionnaires provided a picture of the telecommunication use, pointed to the potential bottlenecks for distant education in Ukraine, mirrored users needs in tools, services, and information support. Many participants expressed a wish to have a kind of hand-book on the Internet, which can be addressed for the particular inquiries, and would contain references for further exploration. This idea was taken into account when working on the STACCIS project.

STACCIS is aimed at dissemination of information about telecommunication applications, tools and techniques in Community of Independent States (CIS) countries through the network of so-called 'information and demonstration centres' (IDC). An IDC contains its own collections and a number of electronic catalogues comprising references to electronic publications, software, tools, services, distance courses and other remote resources. We are currently designing the Basic Internet HyperGlossary as an IDC component. It is intended to assist novices in telecommunications in their search of relevant topics and facilitate professionally-oriented learning.

The Hyper-Glossary will include terms related to the networks, protocols, data interchange devices, search and navigation, and Web-pages design. Its role is two-fold. Firstly it will provide explanation in Russian for terms entered either in English or in Russian. Currently, glossaries in popular editions does not include English terminology which is needed for those users who work in original environments. On the other hand, online Internet glossaries provide explanation of English terms in English only.

Secondly, it will connect separate terms by the context cross-references and will refer to the other sources at the IDC where additional information can be found. The electronic sources intended for instructional purposes (for instance, texts of distance courses) will then be linked back to the HyperGlossary entries. Thus, the Hyper-Glossary will be used both as bilingual glossary and as an explanatory dictionary for study of the Internet-related concepts.

Despite a relatively large amount of the online dictionaries explaining abbreviations and terminology related to the Internet, telecommunications and networks, as well as the existence of other sources oriented to the 
different types of users, the content of the Hyper-Glossary could not be easily compiled. The reason was that in many popular editions the explanation style is oversimplified, the choice of terms is not well-grounded and concepts are explained in isolation. Thus, the Hyper-Glossary creators had to proceed with the whole cycle of the glossary design, including: the choice of concepts; design of the specific concept map representing the relations between the concepts; composition of the definitions and explanations of the concepts; tracing cross-references; ensuring consistency of the glossary as a whole; and arranging references to the additional instructional sources.

The above described design stages will hopefully ensure the quality of the Hyper-Glossary, which is the most critical issue for the emerging digital resources. We also hope that a more rigorous and scholarly style of presentation will correspond to the national traditions in education that ensure the understanding of what an entity is, its connection to other entities, and the overall principles of its functioning. Modern digital sources inform mostly only the usage or purpose. The concept-based approach of information presentation may be preferable for adult people educated according to the local traditions. Moreover, it could diminish a load over memory, and make learning more time-intense, as well as decrease the influence of negative psychological factors revealed in the adult training.

\section{CONCLUSION}

The rapid introduction of technological innovation into educational applications should be accompanied by reconsideration of pedagogical and cognitive importance of proposed novelties. The same way as hyper-books are incorporating features first introduced in research on intelligent tutoring, adaptive interfaces and user modelling (Brusilovsky and Schwarz 1997), VLE should update the functions and features of its components. Discussion of the hyper-glossary role illustrates the idea that there is a large potential of educational applications even within a restricted technological base.

\section{REFERENCES}

Boy, G. (1996) Learning evolution and software agents emergence. In C. Frasson, G. Gauthier, and A. Lesgold. (eds.) Intelligent Tutoring Systems, Lecture Notes in Computer Science 1086, 10-25.

Brusilovsky P. and Schwarz E. (1997) User as student: towards an adaptive interface for advanced Web-based applications. In A. Jameson, C. Paris and C. Tasso (eds.) Proceedings of 6th International Conference on User modelling, Sardinia, Italy, 2nd-5th June 1997. Berlin: Springer Verlag. 
Elliott G.J., Jones E., and Barker, P. (1996) Authoring semantic hypermedia: A concept mapping approach. Proceedings of ED-MEDIA'96 World Conference on Educational Multimedia and Hypermedia. Charlottesville, USA: AACE.

Kommers, P.A.M., Jonassen, D.H. and Mayes, J.T. (1991) Cognitive Tools for Learning; NATO ASI Series Vol. 81. Berlin: Springer Verlag.

Kommers, P. A. M. and Zhiming Zhao. (1998) Conceptual Support with Virtual Reality in Web-based Learning. International Journal of Continuing Engineering Education and Lifelong Learning. 8(1/2), 184-204.

Novak J.D. (1990) Concept mapping: A useful tool for science education. Research in Science Teaching. 27, 937-949.

Sinitsa, K, and Mizoguchi, R. A (1998) Structured hyper-glossary as a cognitive tool for computer-based learning. Proceedings of CATE-98, International Conference on Advanced Technologies in Education. Mexico, 1998.

\section{BIOGRAPHIES}

Both authors, Katherine Sinitsa and Alla Manako, obtained their PhD. Degrees in Computer Science from Institute of Cybernetics and currently are senior researchers in the Dialogue and Tutoring systems Department of IRTC.

Katherine has been engaged in many projects related to computer-based training, authoring systems design, research in the field of intelligent tutoring systems and user modelling. She has worked as a guest researcher in Germany and Osaka University, Japan. Currently her primary interests are focused on tools and environments for lifelong learning, and virtual education. She is also involved in standards activity in the field of information technology terminology in Ukraine and chairs Glossary Working Group in IEEE Learning Technology Standards Committee.

Alla's research interests are data bases, cognitive aspects of courseware creation, multilingual support for education and training, distance learning, and creation of digital resources and repositories. She was the designer of the first Ukrainian distance course on "Basics of the Internet", and the designer and Web-master of the STACCIS Information and Demonstration Centre in Kiev. Currently she is a participant of a COPERNICUS project and is involved in the creation of the Centre of Distance Education which will be designed as a virtual organisation and hosted by IRTC. 